Journal of Animal and Veterinary Advances 11 (16): 2885-2889, 2012

ISSN: $1680-5593$

(C) Medwell Journals, 2012

\title{
The Histological Structure of the Liver and Pancreas and Distribution of Glucagon of Pancreas in the Siberian Tiger (Panthera tigris altaica)
}

\author{
${ }^{1}$ Fugui Fang, ${ }^{2}$ Xiuhong Zhou, ${ }^{1} \mathrm{Fubao} \mathrm{Li},{ }^{3}$ Zhizhong Zhang and ${ }^{1}$ Shudong Jiang \\ ${ }^{1}$ College of Animal Sciences and Technology, Anhui Agricultural University, \\ ${ }^{2}$ Biotechnology Center of Anhui Agricultural University, \\ No. 130 of Changjiang West Road, Hefei, 230036 Anhui, P.R. China \\ ${ }^{3}$ Hefei Wild Animal Park, Hefei, 230061 Anhui, China
}

\begin{abstract}
Glucagon is the hyperglycemic factor that opposes the action of insulin. It stimulates the breakdown of liver glycogen and increased glucogeogenesis. Glucagon is located in the tissues of the pancreas of many animals. However, the distribution of glucag on has not been investigated in the pancreas of the tiger. The present study was performed to describe the histological structure of the liver and pancreas and the location of glucagon of pancreas in the 8 days old Siberian tiger. The tissues of the liver and pancreas were collected from three 8 days old Siberian tigers that died of starvation. The weight was recorded and the histological sections were made and stained with HE. The localization of glucagon in tissue was detected by immunohistochemical two-step PV-9000. The results show that the average weight of the liver and pancreas was approximately 39.06 and $1.97 \mathrm{~g}$, respectively. The tissue structure of the liver consisted of hepatic lobulation and portal area. Central vein, hepatocyte tube and hepatic sinusoid and lymphatic tissue were found in hepatic lobulation. Interlobular veins, interlobular artery and interlobular bile duct were presented in portal area. The pancreas was basically made up of exocrine portion and pancreas islet. The exocrine secretion of the pancreas included many pancreatic acinuses and centroacinar cell was observed in the pancreatic acinus. Glucagon-immunopositive cells were mainly located in the pancreas islet. The current study preliminarily revealed the histological features of the liver and pancreas and the distribution of glucagon in the pancreas of 8 days old Siberian tiger which requires further investigation.
\end{abstract}

Key words: Siberian tiger, liver, pancreas, histological structure, glucagon

\section{INTRODUCTION}

Siberian tiger (Panthera tigris altaica) which is also known as the Amur, Korean, Altaic, North Chinese or Ussuri tiger is a subspecies of tigers. The dual threats of illegal poaching and the destruction of its habitat have brought this species to the brink of extinction with a population of 450 in the world and 20 in China (Hu et al., 2006).

A few studies have reported on tiger oocyte, embryo, genetics and cloning (Gjorret et al., 2002; Hu et al., 2006; Putranto et al., 2007; Liu et al., 2010). However, no studies have identified the histological structure of the liver and pancreas and cytological characteristics of glucagon of pancreas in the Siberian tiger. With the restricted population of the tiger, it would be a challenge for scientists to learn and familiarize themselves with the morphologic characteristics of glucagon in the tiger.
Glucagon is an 29 amino acid peptide. Glucagon restores normal blood glucose levels by increasing hepatic glucose production and disruption of glucagon signalling results in hypoglycemia (Qureshi et al., 2004; Sloop et al., 2004; Lou et al., 2011). Glucagon secretion is suppressed in response to elevated blood glucose levels whereas reduced blood glucose enhances glucagons release. Glucagon is the principal hormone stimulating hepatic glucose production. Previous studies have analyzed the pancreatic development (Manakova and Titlbach, 2007; Chandavar and Naik, 2008; Jeon et al., 2009; Carlsson et al., 2010) and endocrine cells (Yukawa et al., 1999; $\mathrm{Ku}$ et al., 2004; $\mathrm{Ku}$ and Lee, 2006). In addition, it has laso been reported that glucagon is distributed in the pancreatic islet of many animals in a regionally specific pattern in the pancreas (Gustavsen et al., 2008; Huang et al.,

Corresponding Author: Shudong Jiang, College of Animal Sciences and Technology, Anhui Agricultural University, No. 130 of Changjiang West Road, Hefei, 230036 Anhui, P.R. China 
2009; Palazon-Fernandez et al., 2011). However, the regional distribution of glucagon in the pancreas of Siberian tiger remains unclear.

The present study was the first observation of the histological structure of the liver and pancreas and localization of glucagon in the pancreas in the neonatal male Siberian tiger. The results that are presented here will help us understand the biological characteristics of this endangered species.

\section{MATERIALS AND METHODS}

Animals and sample collection: The samples were collected from three male Siberian tigers that died accidentally at the Hefei Wildlife Park in Anhui province in China. No abnormalities were found. The tigers were 8 days of age. After death, the abdominal cavity was opened and the liver and pancreas were dissected and immediately brought to the laboratory in saline at $4^{\circ} \mathrm{C}$. Subsequently, the weight of the liver and pancreas were recorded.

Preparation of sections: A small tissue sample of the liver and pancreas was collected and fixed for $24 \mathrm{~h}$ in $4 \%$ paraformaldehyde (Klinipath, Geel, Belgium) at $4^{\circ} \mathrm{C}$ and after paraffin embedding, $5 \mu \mathrm{m}$ serial sections were prepared, placed on poly-L-lysine-coated glass slides and dried for $1 \mathrm{~h}$ at $60^{\circ} \mathrm{C}$ for histological and immunohistochemical analyses.

Histological evaluation: The sections of each tissue were stained with Hematoxylin and Eosin (HE) for light microscopic examination of the normal histological structure.

Immunohistochemical procedure: Each section was deparaffinized, rehydrated and immunostained by using standard procedures (PV-9000 Method) with rabbit anti-glucagon (1:100 dilution, code BA1619, Zhongshan Goldenbridge Biotechnology Co., Ltd. Beijing, China) as the primary antibody. The secondary antibody was biotinylated goat anti-rabbit IgG (1:100 dilution, Golden Bridge International, Inc., USA). Samples were developed using standard DAB reagents (Zhongshan Goldenbridge Biotechnology Co., Ltd. Beijing, China). After immunostaining, the sections were observed under a light microscope. The negative controls were produced by substituting the primary antibody with $0.01 \mathrm{M}$ PBS.

\section{RESULTS}

The structure of the liver: The average weight of liver was about $39.07 \mathrm{~g}$. The histological structure of the

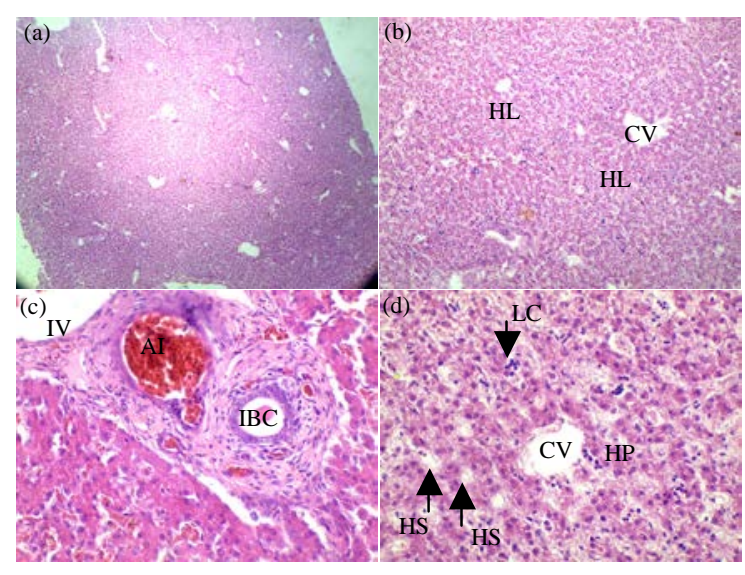

Fig. 1: Histological structure of liver in the 8 days old Siberian tiger using the $\mathrm{HE}$ Method. a) overall liver histological structure, $\mathrm{x} 40$; b) Central Vein (CV) and Hepatic Lobulation (HL), x100; c) Interlobular Vein (IV), Arteriae Interlobulares (AI) and Interlobular Biliary Canals ( $(B C), x 400$ and d) Hepatic Plate (HP), Hepatic Sinusoid (HS) and Lymphocyte (LC), x400

liver consisted of hepatic lobulation and portal area (Fig. 1a). The lobulation was not well defined because the interlobular connective tissue was not developed (Fig. 1b). The central vein presented in the centre of hepatic lobulation, the hepatic plate was radially arranged around the central vein, the reticular vascular between the plate was hepatic sinusoid, the wall of sinusoid formed by endothelial cell. Kupffer cells were found in the sinusoids (Fig. 1d). The hepatic plate was arranged with the single hepatocytes (Fig. 1d). The primary cell in the liver was the hepatocyte. There was polarity of hepatocytes. The portal area consisted of interlobular veins, interlobular artery and interlobular bile duct (Fig. 1c). Lymphatic tissue was found in the lobulation (Fig. 1d).

The structure of the pancreas: The average mass of the pancreas was $1.79 \mathrm{~g}$. The pancreas was divided into many pancreatic lobules by surface connective tissue (Fig. 2a). The pancreas consisted of exocrine portion and endocrine portion. The exocrine secretion of the pancreas was cells which the nuclei was big and round, there are centroacinar cell was found in the narrow acinar lumen (Fig. 2b). The catheter was responsible for carrying pancreatic juice to duodenum. The walls of all catheters were surrounded by a single layer of epithelial cells. The islets were the endocrine portion of the pancreas which the specific cell types could not identity by HE (Fig. $2 \mathrm{c}$ and d).

Location of glucagon in the pancreas: In the pancreas, glucagon-immunoreactive cells were and mainly localized 


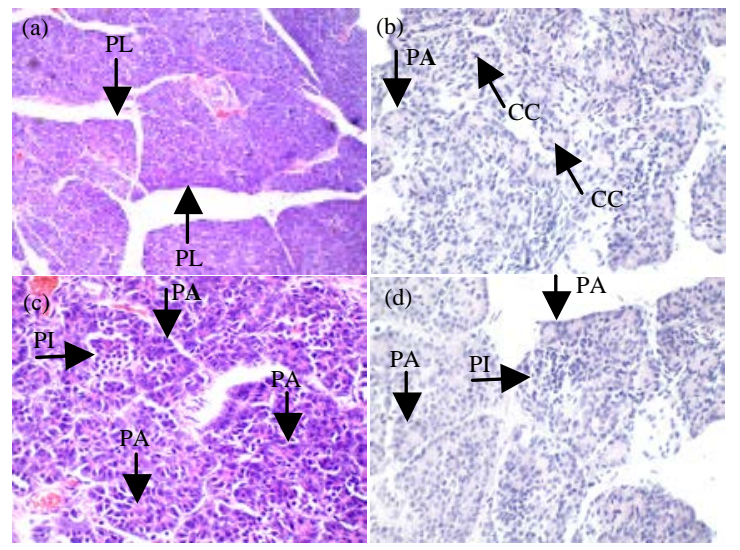

Fig. 2: Histological structure of pancreas in 8 days old Siberian tiger using the HE Method; a) Pancreatic Leaflet (PL), x100; b) Pancreatic Acinar (PA) and Centroacinar Cell (CC), x400; c) Pancreas Islet (PI), $\mathrm{x} 400$ and d) pancreas islet and pancreatic acinar, $\mathrm{x} 400$

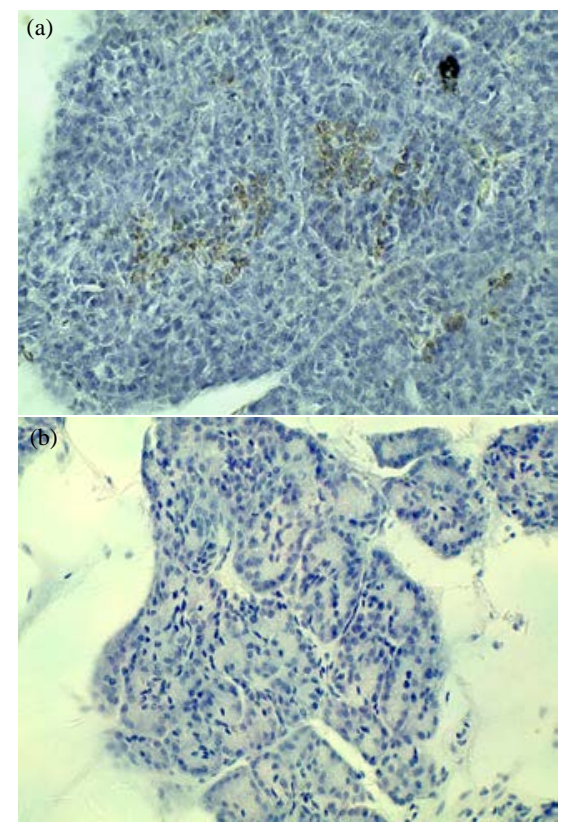

Fig. 3: The distribution of glucagon in the pancreas of the 8 days old Siberian tiger using the PV-9000 Method. Images are shown at the magnification 400; a) immunoreactive cells in the pancreatic islet and $b$ ) the negative control for the pancreas

in the central regions of pancreatic, islet in the exocrine portion, they were randomly scattered in pancreatic acinar cells. Glucagon-immunoreactive cells were round or oval, cytoplasm was deeply stained which cytoplasmic particles clearly visible (Fig. 3a). No glucagon-immunoreactive cells were detected in the pancreatic duct portions. However, glucagon staining was not observed when the glucagon antibody was replaced with PBS (Fig. 3b).

\section{DISCUSSION}

For the first time, the current study showed the structural details in the liver and pancreas and the distribution of glucagon in the pancreasof the 8 days old Siberian tiger.

The hepatic lobulation and portal area were presented in the liver and the lobulation was not well defined because of thin interlobular connective tissue in the Siberian tigers which is the same to the structure of the liver in the cattle, sheep, horse and birds (Peng, 2009).

But interlobular connective tissue in the liver was developed in the pig, cat and camel (Shen, 2002). Abundant lymphocytes were distributed in the hepatic lobulation which is different from other mammals, indicating local immune function is very strong in the liver of the Siberian tigers. It is generally known that the pancreas of vertebrates is subdivided into exocrine and endocrine portions. The pancreas of the Siberian tiger had the same features.

Glucagon is synthesized in the A cells of the pancreas and regulates blood glucose levels ( $\mathrm{Ku}$ et al., 2002a, b). In the present study, glucagon-immunoreactive cells were found in the central regions of pancreatic islets. Although, glucagon-immunoreactive cells have been found in the central regions of equine pancreatic islets (Ku et al., 2002a) including the present study, speciesdependent variations have been reported. Glucagonimmunoreactive cells were found in the mantle and peripheral regions of mammalian pancreatic islets, exocrine portions and pancreatic duct (Krause et al., 1989; Sasaki et al., 1991; da Mota et al., 1992; Edwin et al., 1992; Gomez Dumm et al., 1995; Wieczorek et al., 1998). In addition, it was also reported that under specific disease conditions such as obese (diabetic condition) mouse, glucagon-immunoreactive cells were intermingled with insulin-immunoreactive cells in the central regions of pancreatic islets in contrast, normal non-obese littermates showed a peripheral localization of these immunoreactive cells (Ku et al., 2002b). In the red-bellied frog (Bombina orientalis), glucagon-immunoreactive cells were also detected as single cells or as clusters but in the case of clusters, two distributional patterns were detected-a central core type and a marginally distributed type (Lee et al., 2003). These findings are considered to represent a species-dependent unique distributional pattern. 


\section{CONCLUSION}

Researchers reported for the first time the histological structures of liver and pancreas and the distribution of glucagon in the pancreas of male Siberian tigers aged at 8 days old. However, these results were based on histology. Further research is required to understand the physiology at the cellular and molecular level.

\section{ACKNOWLEDGEMENT}

Researchers acknowledge the funding support from the Natural Science Foundation of the Anhui Education Department (Grant No.: KJ2011A117).

\section{REFERENCES}

Carlsson, G.L., R.S. Heller, P. Serup and P. Hyttel, 2010. Immunohistochemistry of pancreatic development in cattle and pig. Anat. Histol. Embryologia, 39: 107-119.

Chandavar, V.R. andP.R. Naik, 2008. Immunocytochemical detection of glucagon and insulin cells in endocrine pancreas and cyclic disparity of plasma glucose in the turtle Melanochelys trijuga. J. Biosci., 33: 239-247.

Edwin, N., J. Yamada and C.M. Leigh, 1992. A lightmicroscopic immunocytochemical study of the endocrine pancreas in the Australian fat-tailed dunnart (Sminthopsis crassicaudata). Singapore Med. J., 33: 260-261.

Gjorret, J.O., E.G. Crichton, N.M. Loskutoff, D.L. Armstrong and P. Hyttel, 2002. Ultrastructure of oocyte maturation, fertilization and early embryo development in vitro in the Siberian tiger (Panthera tigris altaica). Mol. Reprod. Dev., 63: 79-88.

Gomez Dumm, C.L., G.M. Console, G.C. Luna, M. Dardenne and R.G. Goya, 1995. Quantitative immunohistochemical changes in the endocrine pancreas of nonobese diabetic (NOD) mice. Pancreas, 11: 396-401.

Gustavsen, C.R., N. Pillay and R.S. Heller, 2008. An 1 immunohistochemical study of the endocrine pancreas of the African ice rat, Otomys sloggetti robertsi. Acta Histochem., 110: 294-301.

Hu, X.L., M.Y. Zhu, Z.H. Zhang, R. Hou, F.J. Shen, F.Z. Li and A.J. Zhang, 2006. Cloning, characterization and tissue specific expression of Amur tiger (Panthera tigris altaica) IGF-I. Biosci. Biotech. Bioch., 70: 1846-1854.

Huang, Y.H., M.J. Sun, M. Jiang and B.Y. Fu, 2009. Immunohistochemical localization of glucagon and pancreatic polypeptide on rat endocrine pancreas: Coexistence in rat islet cells. Eur. J. Histochem., 53: 81-85.
Jeon, J., M. Correa-Medina, C. Ricordi, H. Edlund and J.A. Diez, 2009. Endocrine cell clustering during human pancreas development. J. Histochem. Cytochem., 57: 811-824.

Krause, W.J., J.H. 3rd Cutts, J.H. Cutts and J. Yamada, 1989. Immunohistochemical study of the developing endocrine pancreas of the opossum (Didelphis virginiana). Acta Anat., 135: 84-96.

Ku, S.K. and H.S. Lee, 2006. An immunohistochemical study of the pancreatic endocrine cells of the nude mouse, Balb/c-nu/nu. Eur. J. Histochem., 50: 61-68.

$\mathrm{Ku}$, S.K., H.S. Lee and J.H. Lee, 2002a. Immunohistochemical study of the pancreatic endocrine cells in the $\mathrm{BALB} / \mathrm{c}$ mice: An unique distributional pattern of glucagon. J. Vet. Sci., 3: 167-173.

$\mathrm{Ku}$, S.K., H.S. Lee and J.H. Lee, 2004. An immunohistochemical study of gastrointestinal endocrine cells in the BALB/c mouse. Anat. Histol. Embryol., 33: 42-48.

$\mathrm{Ku}$, S.K., H.S. Lee, J.H. Lee, 2002b. An immunohistochemical study on the pancreatic endocrine cells of the C57BL/6 mouse. J. Vet. Sci., 3: $327-333$.

Lee, J.H., S.K. Ku, H.S. Lee and H. Kitagawa, 2003. An immunohistochemical study of endocrine cells in the pancreas of the Red-bellied frog (Bombina orientalis). Eur. J. Histochem., 47: 165-172.

Liu, C.Q., T.F., Feng, B.G. Feng, Liu, W.J., Guan and Y.H. Ma, 2010. Construction of cDNA library and preliminary analysis of expressed sequence tags from Siberian tiger. Int. J. Biol. Sci., 6: 584-589.

Lou, P.H., N. Gustavsson, Y. Wang, G.K. Radda and W. Han, 2011. Increased lipolysis and energy expenditure in a mouse model with severely impaired glucagon secretion. PloS one, Vol. 6.

Manakova, E. and M. Titlbach, 2007. Development of the chick pancreas with regard to estimation of the relative occurrence and growth of endocrine tissue. Anat. Histol. Embryol., 36: 127-134.

Palazon-Fernandez, J.L., M.P. Suso, J.M. Mancera and C. Sarasquete, 2011. Immunohistochemical study of the principal pancreatic islet of the toadfish, Halobatrachus didactylus (Pisces: Batrachoididae). Acta Histochem., 113: 256-261.

Peng, K.M., 2009. Animal Histology and Embryology. China Higher Education Press, Beijing, pp: 150-154.

Putranto, H.D., S. Kusuda, K. Inagaki, G. Kumagai, R. Ishii-Tamura, Y. Uziie and O. Doi, 2007. Ovarian activity and pregnancy in the Siberian tiger, Panthera tigris altaica, assessed by fecal gonadal steroid hormones analyses. J. Vet. Med. Sci., 69: 569-571. 
Qureshi, S.A., M.R. Candelore, D. Xie, X. Yang and L.M. Tota et al., 2004. novel glucagon receptor antagonist inhibits glucagon-mediated biological effects. Diabetes, 53: 3267-3273.

Sasaki, M., T. Arai, T. Usui and T. Oki, 1991. Immunohistochemical, ultrastructural and hormonal studies on the endocrine pancreas of voles (Microtus arvalis) with monosodium aspartateinduced diabetes. Vet. Pathol., 28: 497-505.

Shen, X.F., 2000. Domestic Animal Histology and Embryology. China Agricultural Press, Beijing, pp: 161-167.

Sloop, K.W., J.X.C. Cao, A.M. Siesky, H.Y. Zhang and D.M. Bodenmiller et al., 2004. Hepatic and glucagon-like peptide-1-mediated reversal of diabetes by glucagon receptor antisense oligonucleotide inhibitors. J. Clin. Invest., 113: 1571-1581.
Wieczorek, G., A. Pospischil and E. Perentes, 1998. A comparative immunohistochemical study of pancreatic islets in laboratory animals (rats, dogs, minipigs, nonhuman primates). Exp. Toxicol. Pathol., 50: $151-172$.

Yukawa, M., T. Takeuchi, T. Watanabe and S. Kitamura, 1999. Proportions of various endocrine cells in the pancreatic islets of wood mice (Apodemus speciosus). Anat. Histol. Embryol., 28: 13-16.

da Mota, D.L., J. Yamada, L.L. Gerge and P.B. Pinheiro, 1992. An immunohistochemical study on the pancreatic endocrine cells of the three-toed sloth, Bradypus variegatus. Arch. Histol. Cytol., 55: 203-209. 